\title{
Absent mutations make their presence felt
}

DOI:

$10.1038 /$ nrg2128

URLs

Entrez Links

Smarca5

http://www.ncbi.nlm.nih.gov/

entrez/query.fcgi?db=gene\&c

$\mathrm{md}=$ Retrieve\&dopt=full

reportElist_uids $=93762$

Dnmt1

http://www.ncbi.nlm.nih.gov/ entrez/query.fcgi?db=gene\&c $\mathrm{md}=$ Retrieve\&dopt=full reportElist_uids=13433

Emma Whitelaw's laboratory: http://www.qimr.edu.au/ research/labs/emmaw/index. html
Epigenetic marks are generally thought to be erased as they are passed from parent to offspring. However, a few examples are known in which the slate is not wiped clean and parents pass on the epigenetic state at certain alleles - known as epialleles - to the next generation. A recent study in mice reveals an important implication of such transgenerational epigenetic inheritance: mutations in genes that affect the epigenome can have a phenotypic effect even in offspring that don't inherit the causal genotype.

Chong and colleagues used the mouse agouti viable yellow $\left(A^{v y}\right)$ allele as a reporter for alterations in the inheritance of an epigenetic state. In this epiallele, a transposon has been inserted close to the agouti gene, and the level of expression depends on the DNA-methylation status of the transposon. The extent

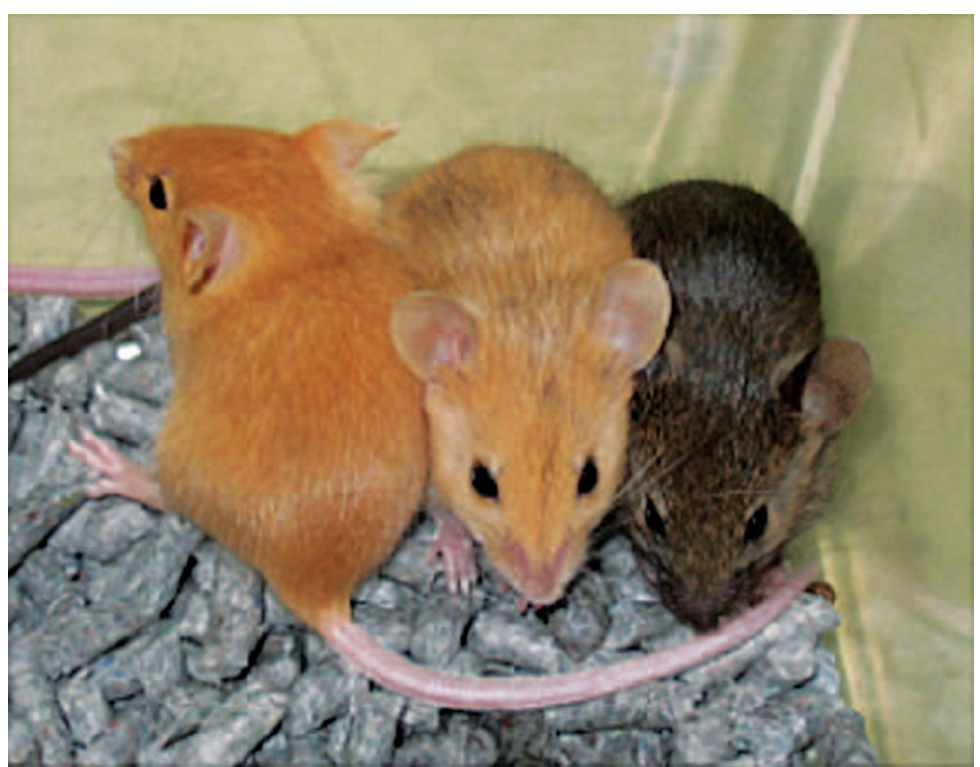

Reproduced from Nature Genetics 39, 614-622 (c) (2007) Macmillan Publishers. of methylation can be gauged through a coat-colour phenotype, with hypomethylation and high levels of expression from $A^{v y}$ producing a yellow coat.

The authors carried out crosses between female mice that carried a single copy of the $A^{v y}$ reporter allele and males that were heterozygous for mutations in one of two genes that are involved in epigenetic reprogramming during early development.

These genes are $S n f 2 h$ (also know as Smarca5), which encodes a member of the ISWI family of ATP-dependent chromatin-remodelling enzymes, and Dnmt1, which encodes a DNA methyltransferase.

An interesting result was seen when offspring from the $S n f 2 h$ cross that were wild type for this gene were compared with offspring from control crosses, in which the maternal genotype was the same

but the male parents were wild type for $S n f 2 h$. Despite having identical genotypes, the two sets of offspring showed a different range of coat colours, with offspring from the Snf $2 h$-heterozygous fathers being more likely to be yellow. As the two groups differed only in the paternal genotype, the authors concluded that the $S n f 2 h$ mutation in the heterozygous fathers must have had an effect on the epigenome such that it alters the epigenetic state at $A^{v y}$. A similar result was seen when the equivalent comparison was made for offspring from the Dnmt1 cross. These are the first described examples in mammals of paternal effects, in which the untransmitted genotype of the father has an effect on the phenotype of the offspring.

An important challenge now is to understand how these paternal effects are exerted at the molecular level. For example, $S n f 2 h$ is expressed in the male germ line, but how the maternally inherited $A^{v y}$ allele is affected by untransmitted mutations in this gene is currently unclear. However, it is clear that paternal effects (and potentially maternal effects) that influence the epigenome need to be explored as a source of phenotypic variation in mammals. In particular, investigating whether similar phenomena occur in humans might lead to new insights into mechanisms that underlie disease. Louisa Flintoft

ORIGINAL RESEARCH PAPER Chong, S. et al Modifiers of epigenetic reprogramming show paternal effects in the mouse. Nature Genet. 39 , 614-622 (2007)

WEB SITE

Emma Whitelaw's laboratory: http://www.qim edu.au/research/labs/emmaw/index.htm 